\title{
Correction to: The discovery of novel predictive biomarkers and early-stage pathophysiology for the transition from gestational diabetes to type 2 diabetes
}

\author{
Saifur R. Khan ${ }^{1,2} \cdot$ Haneesha Mohan ${ }^{1,2} \cdot$ Ying Liu $^{1,2} \cdot$ Battsetseg Batchuluun $^{1,2} \cdot$ Himaben Gohil $^{1,2}$ - Dana Al Rijjal ${ }^{1,2}$. \\ Yousef Manialawy ${ }^{1,2} \cdot$ Brian J. Cox ${ }^{3,4}$. Erica P. Gunderson ${ }^{5}$ - Michael B. Wheeler ${ }^{1,2}$
}

Published online: 8 February 2019

(C) Springer-Verlag GmbH Germany, part of Springer Nature 2019

\section{Correction to: Diabetologia}

https://doi.org/10.1007/s00125-018-4800-2

Unfortunately, the graph in Fig. $5 \mathrm{f}$ became misaligned during typesetting. The corrected version of Fig. 5 is reproduced here.

The online version of the original article can be found at https://oi.org/ $10.1007 / \mathrm{s} 00125-018-4800-2$

Brian J. Cox

b.cox@utoronto.ca

$\triangle$ Erica P. Gunderson

erica.gunderson@kp.org

$\triangle$ Michael B. Wheeler

michael.wheeler@utoronto.ca

1 Endocrine and Diabetes Platform, Department of Physiology,

University of Toronto, Medical Sciences Building, Room 3352, 1

King's College Circle, Toronto, ON M5S 1A8, Canada
2 Advanced Diagnostics, Metabolism, Toronto General Hospital Research Institute, Toronto, ON, Canada

3 Reproduction and Development Platform, Department of Physiology, University of Toronto, Medical Sciences Building, Room 3360, 1 King's College Circle, Toronto, ON M5S 1A8, Canada

4 Department of Obstetrics and Gynecology, University of Toronto, Toronto, ON, Canada

Kaiser Permanente Northern California, Division of Research, 2000 Broadway, Oakland, CA 94612, USA 
a $\quad$ b

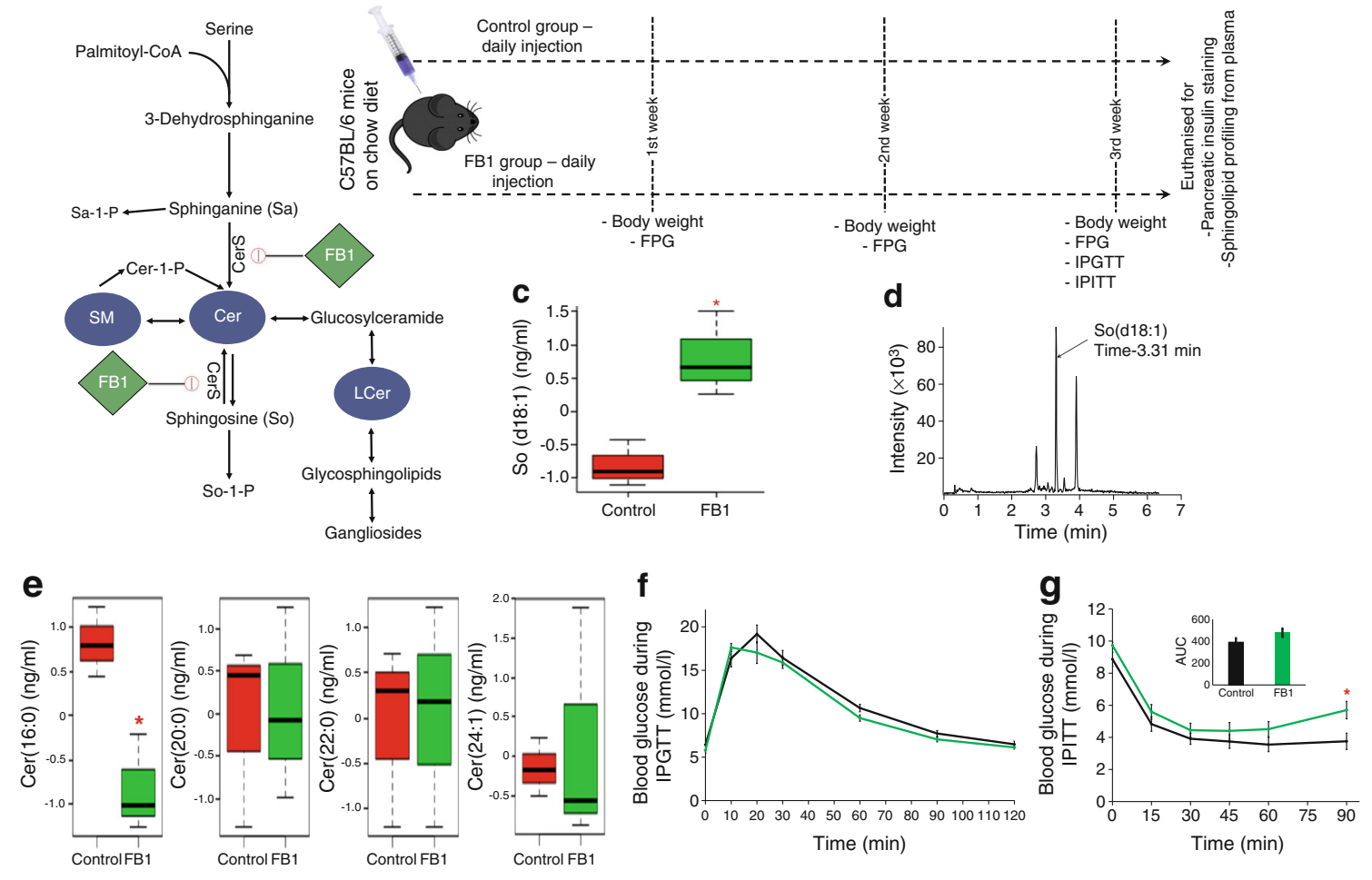

h
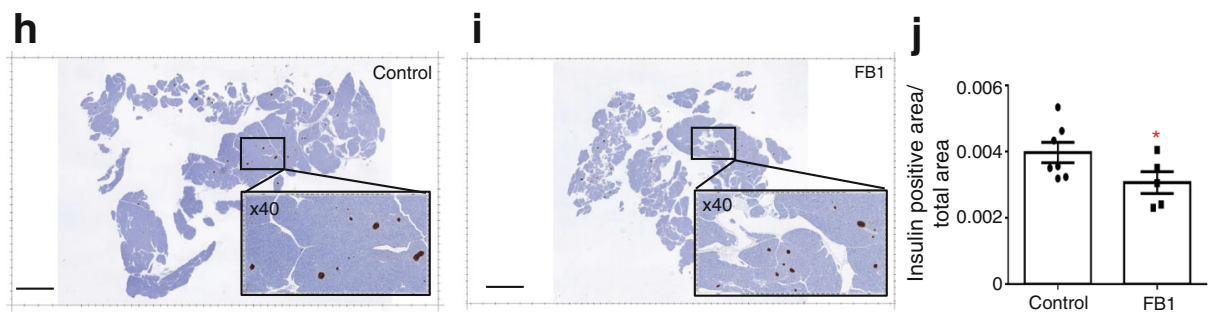

Fig. 5 In vivo functional studies. (a) Schematic flow diagram of the sphingolipid metabolism pathway showing targets of FB1 (pharmacological inhibitor). (b) The in vivo study design ( $n \geq 14)$ : the control group of mice was injected with vehicle while the treatment group was injected with FB1 (1 mg/kg) daily. Every week, the weight gain and the FPG were monitored. At the end of the third week, GTT and ITT were performed. Finally, all mice were euthanised to collect whole pancreases and plasma. (c) So concentration in control and FB1-treated mice $(n=3)$. (d) Representative chromatogram of So. (e) Comparison of the four Cer species found to significantly differ in the SWIFT cohort (values were mean-centred $[n=3]$ and divided by the SD of each variable). In the boxplots (c, e), the bottom and top of the box are the Q1 and Q3 (25th and 75th percentile), respectively, and the central band is the median (Q2 or 50th percentile). The bottom whisker is located within $1.5 \mathrm{IQR}$ of the lower quartile, and the upper whisker is located within 1.5 IQR of the upper quartile. (f) GTT single time point comparison between control (black line) and FB1 group (green line) at the end of 3 weeks treatment $(n \geq 7)$. (g) ITT single time point comparison between control (black line) and FB1 group (green line) at the end of 3 weeks treatment $(n \geq 7)$; inset shows AUC (mmol/1 $\times$ min). (h, i) Representative insulin-stained pancreas (5 $\mu \mathrm{m}$ thickness, longitudinally sectioned through the pancreatic headto-tail axis) from control (h) and FB1-treated mice (i); scale bars, $3 \mathrm{~mm}$; insets show $\times 40$ magnification. (j) Insulin-positive area in pancreases of control and FB1-treated mice $(n \geq 5)$. A two-tailed, unpaired $t$ test was carried out for each comparison. Data are presented as mean $\pm \mathrm{SEM}$; unadjusted $p$ values: ${ }^{*} p<0.05$ vs control

Publisher's note Springer Nature remains neutral with regard to jurisdictional claims in published maps and institutional affiliations. 